\title{
Geometric scalar theory of gravity
}

\author{
M. Novello ${ }^{1 *}$, E. Bittencourt ${ }^{1}$, U. Moschella ${ }^{2}$, E. Goulart ${ }^{1}$, J. M. Salim ${ }^{1}$, and J. Toniato ${ }^{1}$ \\ ${ }^{1}$ Instituto de Cosmologia Relatividade Astrofisica ICRA - CBPF \\ Rua Dr. Xavier Sigaud 150 - 22290-180 Rio de Janeiro - Brazil \\ and \\ ${ }^{2}$ Università degli Studi dell'Insubria - Dipartamento di Fisica e Matematica \\ Via Valleggio 11 - 22100 Como - Italy and \\ INFN, Sez di Milano, Via Celoria 16, \\ 20146, Milano - Italy
}

(Dated: June 4, 2018)

\begin{abstract}
We present a geometric scalar theory of gravity. Our proposal will be described using the "background field method" introduced by Gupta, Feynman, Deser and others as a field theory formulation of general relativity. We analyze previous criticisms against scalar gravity and show how the present proposal avoids these difficulties. This concerns not only the theoretical complaints but also those related to observations. In particular, we show that the widespread belief of the conjecture that the source of scalar gravity must be the trace of the energy-momentum tensor-which is one of the main difficulties to couple gravity with electromagnetic phenomenon in previous models - does not apply to our geometric scalar theory. Some consequences of the new scalar theory are explored.
\end{abstract}

PACS numbers: 04.20.-q, 11.10.-z

\section{INTRODUCTION}

In recent years a few papers have appeared reviewing the scalar theory of gravity and pointing out the reasons for which that theory was dismissed (see [1], 2] and [3] and references therein for a fairly complete review on scalar theories of gravity). Criticisms range from theoretical to observational; they are based on the hypothesis made since the old days of the Einstein-Grosmann proposal [4] that scalar gravity deals with essentially three assumptions:

- The theory is described in a conformally flat geometry and the background Minkowski metric is observable;

- The source of the gravitational field is the trace of the energy-momentum tensor;

- The scalar field is the (special) relativistic generalization of the Newtonian potential.

In this paper we present a new possibility for describing the gravitational interaction in terms of a scalar field $\Phi$, i.e. a geometric scalar gravity (GSG). The above three assumptions do not hold in our theory. In particular, we shall see that the general belief that the only possible source of scalar gravity is the trace of the energymomentum tensor is not valid here.

Let us clarify from the very beginning that this is not a special relativistic scalar gravity. The adjective geometric in the title pinpoints its similarity with general relativity: we are constructing a metric theory of gravity.

\footnotetext{
*M. Novello is Cesare Lattes ICRANet Professor
}

In other words, we follow the main idea of general relativity and assume as an a priori that gravity is described by a Riemannian geometry. In general relativity the ten components of the metric tensor are the basic variables of the theory (up to coordinate transformations). Here the metric tensor is determined by the derivatives of a fundamental independent physical quantity represented by the scalar field $\Phi$.

This means that although we make use of a scalar field to represent all gravitational processes we do not follow the previous examples of scalar gravity, as for instance the Einstein-Grossman "Entwurf Theory". Before presenting the reasons that motivated us to undertake this proposal, let us summarize the main properties of the GSG:

- The gravitational interaction is described by a scalar field $\Phi$;

- The field $\Phi$ satisfies a nonlinear dynamics;

- The theory satisfies the principle of general covariance. In other words, this is not a theory restricted to the realm of special relativity;

- All kind of matter and energy interact with $\Phi$ only through the pseudo-Riemannian metric

$$
q_{\mu \nu}=a \eta_{\mu \nu}+b \partial_{\mu} \Phi \partial_{\nu} \Phi
$$

- Test particles follow geodesics relative to the gravitational metric $q_{\mu \nu}$;

- $\Phi$ is related in a nontrivial way with the Newtonian potential $\Phi_{N}$;

- Electromagnetic waves propagate along null geodesics relative to the metric $q^{\mu \nu}$. 
The parameters $a$ and $b$ are functionals of the scalar field $\Phi$ (which will be specified by fixing the Lagrangian of the scalar field). The auxiliary (Minkowski) metric $\eta^{\mu \nu}$ is unobservable because the gravitational field couples to matter only through $q^{\mu \nu}$. Here we follow the main steps of general relativity where a unique geometrical entity interacts with all forms of matter and energy and the geometry underlying all events is controlled by the gravitational phenomena.

From this postulate it follows immediately that the space-time geometry is an evolutionary process identified to the dynamics of the gravitational field. This beautiful hypothesis of general relativity is contained in each observation as a specific example of a geometry solving that dynamics.

The second important postulate of general relativity states that the metric couples universally and minimally to all fields of the standard model by replacing everywhere the Minkowski metric $\eta_{\mu \nu}$ by $g_{\mu \nu}$.

We will accept also this postulate, but we investigate a special form for the Riemannian metric that represents the gravitational field. We shall describe the origin of this departure from general relativity in a later section after reviewing in the next section the case of general relativity.

A final remark concerns our method to identify the right form of the dynamics of the scalar field. The action of a given theory is generally constructed using certain a priori principles and rules. For instance one might want to impose general covariance, symmetry principles such as the gauge principle and a limitation on the order of derivatives. Although these principles are preserved in our theory they are of course not enough. Here we adopt observation-oriented procedure and try to determine the form of the dynamics of our GSG as a power expansion in the field $\Phi$ whose coefficients are fixed from observations. This means that we try to proceed step by step, adding new terms to the Lagrangian when observation imposes this, and we continue such procedure until the theory achieves its final form. In the following we present an example of how this strategy can be used by the analysis of the motion of test particles (like planetary orbits) in the field generated by a massive body like the sun in the quasi-linear regime.

\section{THE DESCRIPTION OF GENERAL RELATIVITY AS A FIELD THEORY}

Although general relativity is usually presented in the framework of Riemannian geometry, it is possible to fully describe the exact Einstein's theory of gravity in terms of a spin-2 field propagating in an arbitrary background space-time. The case of Minkowskian background was investigated by many authors (see details in [5] and [6]) and for a generic background including Minkowski it was described in 7] and [8].

The main idea can be summarized as follows. Con- sider a flat Minkowski background (just to simplify our exposition) endowed with a metric $\eta_{\mu \nu}$. In a Lorentzian coordinate system the metric of the background takes the standard constant expression. We may allow for general coordinates; the curvature tensor however vanishes:

$$
R_{\beta \mu \nu}^{\alpha}\left(\eta_{\varepsilon \sigma}\right)=0 .
$$

In a galilean coordinate system the metric of the background can assume standard constant expression. From now on $\eta_{\mu \nu}$ are the component (not necessarily constant) of the Minkowski metric in general coordinates. Then one introduces a symmetric second order tensor $h_{\mu \nu}$ and writes

$$
g^{\mu \nu} \equiv \eta^{\mu \nu}+h^{\mu \nu} .
$$

This binomial form is an exact expression for the metric $g^{\mu \nu}$. Note however that its inverse, the covariant tensor $g_{\mu \nu}$ is not in general a binomial form but instead is an infinite series:

$$
g_{\mu \nu}=\eta_{\mu \nu}-h_{\mu \nu}+h_{\mu \alpha} h_{\nu}^{\alpha}+\ldots
$$

There are two main postulates founding general relativity:

- The background Minkowski metric is not observable. Matter and energy interact gravitationally only through the combination $\eta^{\mu \nu}+h^{\mu \nu}$ and its derivatives. Any test body in a gravitational field moves along a geodesic relative to the metric $g_{\mu \nu}$;

- The dynamics of gravity is described by an equation relating the contracted curvature tensor $R_{\mu \nu}$ to the stress-energy tensor of matter.

The scalar theory of gravity that we present here deals with a modification of the flat Minkowski metric similar to the general relativistic one. However, there is a very important distinction concerning the origin of the curvature of the space-time and its dynamics. In our model the (binomial) form of the metric $q_{\mu \nu}$ comes from a principle that we shall now explain.

\section{THE BIRTH OF GEOMETRY IN SCALAR GRAVITY}

In this section we will show that a metric $q_{\mu \nu}$ naturally appears in certain nonlinear scalar field theories. Let us start by considering the following nonlinear Lagrangian in flat Minkowski spacetime:

$$
L=V(\Phi) w,
$$

where $w \equiv \eta^{\mu \nu} \partial_{\mu} \Phi \partial_{\nu} \Phi$. For $V=1 / 2$ this is just the standard free massless Klein-Gordon scalar field. In the general case the usual kinetic term is rescaled by a field 
dependent amplitude (potential) $V(\Phi)$. Here we are using $\eta^{\mu \nu}$ but we could have used an arbitrary coordinate system as well, since the theory is generally covariant and there is no privileged reference frame. The field equation is

$$
\frac{1}{\sqrt{-\eta}} \partial_{\mu}\left(\sqrt{-\eta} \eta^{\mu \nu} \partial_{\nu} \Phi\right)+\frac{1}{2} \frac{V^{\prime}}{V} w=0,
$$

where $V^{\prime} \equiv d V / d \Phi$ and $\eta$ is the determinant of $\eta_{\mu \nu}$.

Now comes a remarkable result: the above field equation (4) can be seen as that of a massless Klein-Gordon field propagating in a curved space-time whose geometry is governed by $\Phi$ itself. In other words, the same dynamics can be written either in a Minkowski background or in another geometry constructed in terms of the scalar field. Following the steps established in [9], we introduce the contravariant metric tensor $q^{\mu \nu}$ by the binomial formula

$$
q^{\mu \nu}=\alpha \eta^{\mu \nu}+\frac{\beta}{w} \partial^{\mu} \Phi \partial^{\nu} \Phi,
$$

where $\partial^{\mu} \Phi \equiv \eta^{\mu \nu} \partial_{\nu} \Phi$ and parameters $\alpha$ and $\beta$ are dimensionless functions ${ }^{1}$ of $\Phi$. The corresponding covariant expression, defined as the inverse $q_{\mu \nu} q^{\nu \lambda}=\delta_{\mu}^{\lambda}$, is also a binomial expression ${ }^{2}$ :

$$
q_{\mu \nu}=\frac{1}{\alpha} \eta_{\mu \nu}-\frac{\beta}{\alpha(\alpha+\beta) w} \partial_{\mu} \Phi \partial_{\nu} \Phi .
$$

Now we ask whether it is possible to find $\alpha$ and $\beta$, in such a way that the dynamics of the field (4) takes the form

$$
\square \Phi=0,
$$

where $\square$ is the Laplace-Beltrami operator relative to the metric $q_{\mu \nu}$, that is

$$
\square \Phi \equiv \frac{1}{\sqrt{-q}} \partial_{\mu}\left(\sqrt{-q} q^{\mu \nu} \partial_{\nu} \Phi\right) .
$$

To answer this question let us evaluate the determinant of the metric $q=\operatorname{det} q_{\mu \nu}$. A direct calculation yields

$$
\sqrt{-q}=\frac{\sqrt{-\eta}}{\alpha \sqrt{\alpha(\alpha+\beta)}}
$$

Using equation (5), it follows that

$$
q^{\mu \nu} \partial_{\nu} \Phi=(\alpha+\beta) \eta^{\mu \nu} \partial_{\nu} \Phi .
$$

The final result is summarized in the following:

\footnotetext{
${ }^{1}$ Note that the quantity $w$ can be written in terms of its corresponding

$$
\Omega \equiv \partial_{\mu} \Phi \partial_{\nu} \Phi q^{\mu \nu}
$$

once one fixes the theory by specifying the Lagrangian. Indeed, we have

$$
\Omega=(\alpha+\beta) w .
$$

From this expression, giving $\alpha$ and $\beta$ we obtain $\Omega$ as function of $w$ and $\Phi$.

2 A metric of this form is also relevant in another context. See for instance [10].
}

Lemma. Given the Lagrangian $L=V(\Phi) w$ with an arbitrary potential $V(\Phi)$, the field theory satisfying Eq. (4) in Minkowski spacetime is equivalent to a massless KleinGordon field $\square \Phi=0$ in the metric $q^{\mu \nu}$ provided that the functions $\alpha(\Phi)$ and $\beta(\Phi)$ satisfy the condition

$$
\alpha+\beta=\alpha^{3} V .
$$

Remarkable, this equivalence is valid for any dynamics described in the Minkowski background by the Lagrangian $L$. This fact can be extended to other kinds of nonlinear Lagrangian (see details in [1] $)$.

\section{THE GENERAL METRIC PRESCRIPTION: TOWARDS THE GEOMETRIZATION OF THE SCALAR GRAVITY}

We have shown how a nonlinear theory based on the Lagrangian $L$ selects a class of metric tensors associated with the dynamics of the scalar field. Is such property just a simple mathematical curiosity or is the previous lemma pointing towards a more ambitious program? Could the associated metric $q^{\mu \nu}$ play a more fundamental role? The following assumption takes a step in the direction of constructing a theory for the gravitational interaction based on the scalar field $\Phi$.

Fundamental Hypothesis. The gravitational interaction is mediated by the scalar field $\Phi$. All forms of matter and energy interact with $\Phi$ only through the metric $q_{\mu \nu}$ and its derivatives in a covariant way.

In the rest of the paper we explore this hypothesis to see whether it can stand not only from the formal side but also in comparison with the observations; we will in particular examine the situation concerning the classical tests of general relativity, cosmology and the gravitational radiation.

It is worthwhile to point out that the scalar field is not the (special) relativistic generalization of the Newtonian potential. Indeed, following the scheme of general relativity [12] and assuming that the test particles follow geodesics relative to the geometry $q_{\mu \nu}$, we have that

$$
\frac{d^{2} x^{i}}{d t^{2}}=-\Gamma_{00}^{i}=-\partial^{i} \Phi_{N}
$$

where we are assuming static weak field configuration and low velocities for test particles.

From Eq. (6), we have

$$
\Gamma_{00}^{i} \approx-\frac{1}{2} \partial^{i} \ln \alpha
$$

It follows that the Newtonian potential $\Phi_{N}$ is approximately given by

$$
\Phi_{N} \approx-\frac{1}{2} \ln \alpha
$$


which yields the relation between the $q-$ metric and Newtonian potential $\Phi_{N}$ as

$$
q_{00}=\frac{1}{\alpha} \approx 1+2 \Phi_{N}
$$

Using equation (7) one obtains the right (vacuum) Newtonian limit:

$$
\nabla^{2} \Phi_{N}=0
$$

This was the starting point of Einstein's path in building his tensorial theory of gravity. The geometric scalar gravity follows another path that we describe next. From now on we will explore the consequences of extrapolating from the above approximation the general expression

$$
\alpha=e^{-2 \Phi}
$$

The next task is to determine the functional dependence of $\beta$ on $\Phi$ or either the form of the potential $V(\Phi)$ once

$$
\beta=\alpha\left(\alpha^{2} V-1\right)
$$

Before doing this, a few comments on previous versions of scalar theories are in order.

\section{THE DIFFICULTIES OF PREVIOUS SCALAR THEORIES OF GRAVITY}

One of the main drawbacks of the ancient proposals for scalar gravity originates from the relation between the field $\Phi$ and Newtonian potential $\Phi_{N}$, while trying to generalize Poisson's equation [2]. Nordström was the first who tried a special relativistic generalization of Newtons gravity [13]. He made the simplest assumption by imposing

$$
\Phi=-4 \pi G \rho,
$$

where $\rho$ is the matter density. Unfortunately the inertial mass of the test particles was no longer constant and the theory could not be derived from a variational principle. Afterwards, Einstein himself [4] stated in a very clear way the possibly right generalization by writing the equation

$$
\Phi=-\kappa T,
$$

where $T$ is the trace of the energy-momentum tensor. In this theory, test bodies move according with the equation

$$
\frac{d}{d \tau}\left(m\left(x^{\alpha}(\tau)\right) v^{\mu}(\tau)\right)=m\left(x^{\alpha}(\tau)\right) \eta^{\mu \nu} \partial_{\nu} \Phi\left(x^{\alpha}(\tau)\right)
$$

where $\tau$ is the proper time. Note that there is a spacetime dependent mass $m$ provided by

$$
m=m_{0} e^{\left(\Phi-\Phi_{0}\right)},
$$

where $m_{0}$ and $\Phi_{0}$ are constant. Einstein's improved theory has an action principle and the equivalence principle holds. However the electromagnetic field does not couple to gravity. In these lines, the best proposal was Einstein and Fokker's reformulation of Nordström theory, in which they set

$$
R=24 \pi G T \text {. }
$$

where $R$ is the curvature scalar. Yet, the electromagnetic field remains uncoupled to the gravitational field. Here is a summary of the main drawbacks of the above proposals:

- Existence of a preferred-frame: All forms of special relativistic theories accept this hypothesis from the very beginning.

- The source of scalar gravity is the trace of the energy-momentum tensor: this originates the main handicap of all previous scalar gravity: gravity does not couple to the electromagnetic field.

- The scalar gravity is conformally flat: Minkowski background is observable.

Even after the advent of General Relativity and its successes in accounting for observations, some alternative theories involving scalar fields in different scenarios have been suggested up to now, aiming to be competitive in explaining the observational tests and sometimes bringing in new physics (see [1, 2, 14]).

Our scalar theory of gravity overcomes the problems we mentioned above: the structure of the metric tensor (5) is covariant from the beginning, the field theory formulation makes the background metric unobservable and the action principle enables a full energy-momentum tensor coupling. It is also worth to emphasize the hypothesis that all bodies move along geodesics relative to the metric

$$
q^{\mu \nu}=\alpha \eta^{\mu \nu}+\frac{\beta}{w} \partial^{\mu} \Phi \partial^{\nu} \Phi
$$

As a realization of this procedure, in the next section we shall see how the electromagnetic fields couple to the gravitational field only through the metric $q_{\mu \nu}$.

\section{ELECTROMAGNETIC FIELD}

The equivalence principle states that all kind of matter and energy interacts with the gravitational field trough minimal coupling with the metric $q_{\mu \nu}$. The old-fashioned scalar gravity models assume that the scalar field only generates a conformally flat metric and that it couples 
to the trace of the energy-momentum tensor. These hypotheses are incorrect. In our geometric scalar gravity (GSG) the electromagnetic field interacts with the scalar field through the metric $q_{\mu \nu}$ as all other kind of matter and energy.

Accordingly the electromagnetic part of the Lagrangian is given by

$$
L=F_{\alpha \mu} F_{\beta \nu} q^{\mu \nu} q^{\alpha \beta} .
$$

The corresponding field equation obtained by the variational principle

$$
\delta \int \sqrt{-q} d^{4} x L=0
$$

is given by

$$
F^{\mu \nu} ; \nu=0
$$

where $F^{\mu \nu} \equiv F_{\alpha \beta} q^{\alpha \mu} q^{\beta \nu}$. The semicolon here represents the covariant derivative w.r.t. the metric $q_{\alpha \beta}$. The Hadamard conditions on discontinuities (see Appendix B) provide the dispersion relation

$$
k_{\mu} k_{\nu} q^{\mu \nu}=0
$$

where $k_{\mu} \equiv \partial_{\mu} \Sigma$ is the gradient of the function $\Sigma$ that defines the surface of discontinuity. Thus electromagnetic waves propagate along null geodesics of the geometry $q_{\mu \nu}$.

We will now complete the theory by specifying parameters $\alpha$ and $\beta$ as functions of $\Phi$. Following the strategy we have choose we have to look into observational conditions. This is made first of all by looking into the gravitational field of spherical symmetric objects.

\section{THE DYNAMICS OF SCALAR GRAVITY}

The usual starting point to build a theory consists in writing an action being guided by certain principles, such as general covariance, second order differential equations, the right Newtonian limit, and so on. A physical theory should however be falsifiable by its observational consequences. Our geometric scalar gravity contains two major ingredients: the interaction of the gravitational field $\Phi$ with matter and all kind of energy through the metric tensor $q_{\mu \nu}$ and the dynamics of $\Phi$ related to the Lagrangian

$$
L=V(\Phi) w
$$

written in the auxiliary (non-observable) Minkowski background. To select among all possible Lagrangians of the above form we look for indications from the various circumstances in which reliable experiments have been performed. In this vein, we initiate the discussion by analyzing the consequences of GSG for the solar system. This is of course not enough, and to fully specify the dynamics we will need to look for further properties. The analysis of planetary orbits allows however to put constraints on the theory as we will now explain.

\section{The static and spherically symmetric solution}

Any theory of gravity must account for planetary orbits. In general relativity this motion is described by geodesics of the Schwarzschild geometry. In the GSG particles follow geodesics in the $q_{\mu \nu}$ metric.

Let us start by rewriting the (unobservable) auxiliary Minkowski background metric in spherical coordinates

$$
d s_{M}^{2}=d t^{2}-d R^{2}-R^{2} d \Omega^{2} .
$$

Changing the radial coordinate to $R=\sqrt{\alpha} r$, where $\alpha=$ $\alpha(r)$ we get

$$
d s_{M}^{2}=d t^{2}-\alpha\left(\frac{1}{2 \alpha} \frac{d \alpha}{d r} r+1\right)^{2} d r^{2}-\alpha r^{2} d \Omega^{2}
$$

Since we are looking for static spherically symmetric solution we assume that the field depends only on the radial variable $\Phi=\Phi(r)$. Then the gravitational metric (6) takes the form

$$
d s^{2}=\frac{1}{\alpha} d t^{2}-B d r^{2}-r^{2} d \Omega^{2},
$$

where we have defined

$$
B \equiv \frac{\alpha}{\alpha+\beta}\left(\frac{1}{2 \alpha} \frac{d \alpha}{d r} r+1\right)^{2}
$$

The field equation (7) then reduces to

$$
\frac{r^{2} \sqrt{\alpha+\beta}}{\alpha}\left(\frac{1}{2 \alpha} \frac{d \alpha}{d r} r+1\right)^{-1} \frac{d \Phi}{d r}=\Phi_{0},
$$

where $\Phi_{0}$ is a constant. Noting that $\alpha(\Phi)=e^{-2 \Phi}$ we proceed by successive approximations (see details in Appendix C) and get an ansatz for the form of the potential $V$ as follows:

$$
V(\Phi)=\frac{(\alpha-3)^{2}}{4 \alpha^{3}} .
$$

By substituting Eqs. (12) and (23) into Eq. (22) gives

$$
e^{2 \Phi} \frac{d \Phi}{d r}=\frac{\Phi_{0}}{r^{2}}
$$

and therefore

$$
\Phi=\frac{1}{2} \ln \left(2 c_{1}-2 \frac{\Phi_{0}}{r}\right),
$$

where $c_{1}$ is an integration constant. The asymptotic behavior implies that $c_{1}=1 / 2$ and $\Phi_{0}=M G / c^{2}$, where $M$ is the mass of gravitational source, $G$ is Newton's constant and $c$ the speed of light, i.e.

$$
\Phi=\frac{1}{2} \ln \left(1-\frac{r_{H}}{r}\right)
$$

where $r_{H} \equiv 2 M G / c^{2}$. The scalar field $\Phi$ reduces to Newtonian potential in the weak field limit. Using $\alpha$ and $\beta$ 
given by (12) and (13), respectively, the line element can be written as

$$
d s^{2}=\left(1-\frac{r_{H}}{r}\right) d t^{2}-\left(1-\frac{r_{H}}{r}\right)^{-1} d r^{2}-r^{2} d \Omega^{2} .
$$

This geometry has the same form as in general relativity and yields the observed regime for solar tests. Thus, the present geometric scalar gravity is a good description of planetary orbits and also for light rays trajectories that follow geodesics (time-like and null-like, respectively) in the $q_{\mu \nu}$ geometry. If new observations would require a modification of the metric in the neighborhood of a massive body this should be made by adjusting the form of the potential $V(\Phi)$.

\section{ACTION PRINCIPLE}

Now that we have found a possible potential for the scalar field we are in position to write its dynamical equations. Let us start by the action written in the auxiliary unobservable Minkowski background. From variational principle

$$
\delta S_{1}=\delta \int \sqrt{-\eta} d^{4} x L
$$

we get:

$$
\delta S_{1}=-\int \sqrt{-\eta} d^{4} x\left(V^{\prime} w+2 V \square_{M} \Phi\right) \delta \Phi
$$

where

$$
\square_{M} \Phi \equiv \frac{1}{\sqrt{-\eta}} \partial_{\mu}\left(\sqrt{-\eta} \eta^{\mu \nu} \partial_{\nu} \Phi\right)
$$

is the d'Alembert operator in flat space (in general coordinates). Using the bridge relations to pass to curved space-time we get

$$
\delta S_{1}=-2 \int \sqrt{-q} d^{4} x \sqrt{V} \square \Phi \delta \Phi .
$$

In presence of matter we add a corresponding term $L_{m}$ to the total action:

$$
S_{m}=\int \sqrt{-q} d^{4} x L_{m}
$$

The first variation of this term as usual yields

$$
\delta S_{m}=-\frac{1}{2} \int \sqrt{-q} d^{4} x T^{\mu \nu} \delta q_{\mu \nu},
$$

where we have defined the energy-momentum tensor in the standard way

$$
T_{\mu \nu} \equiv \frac{2}{\sqrt{-q}} \frac{\delta\left(\sqrt{-q} L_{m}\right)}{\delta q^{\mu \nu}} .
$$

General covariance leads to conservation of the energymomentum tensor $T_{; \nu}^{\mu \nu}=0$. The equation of motion is obtained by the action principle

$$
\delta S_{1}+\delta S_{m}=0 .
$$

Up to this point we are following the paths of general relativity. Here however, in the GSG theory, the metric $q_{\mu \nu}$ is not the fundamental quantity. We have to write the variation $\delta q_{\mu \nu}$ as function of $\delta \Phi$ :

$$
\delta q_{\mu \nu}=\delta\left(\frac{1}{\alpha} \eta_{\mu \nu}-\frac{\beta}{\alpha Z w} \partial_{\mu} \Phi \partial_{\nu} \Phi\right) .
$$

While we are using the unobservable background Minkowski metric for simplicity, at the end all expressions should be written in terms of the gravitational metric $q_{\mu \nu}$. After some calculation we get

$$
\frac{\delta S_{m}}{\delta \Phi}=-\frac{1}{2} \int \sqrt{-q} d^{4} x\left(\frac{\alpha^{\prime}}{\alpha}(E-T)-\frac{Z^{\prime}}{Z} E+2 \nabla_{\lambda} C^{\lambda}\right)
$$

where $Z \equiv \alpha+\beta=\alpha^{3} V$. We have also denoted

$$
T \equiv T^{\mu \nu} q_{\mu \nu}, \quad E \equiv \frac{T^{\mu \nu} \partial_{\mu} \Phi \partial_{\nu} \Phi}{\Omega}, \quad X^{\prime} \equiv \frac{d X}{d \Phi},
$$

and

$$
C^{\lambda} \equiv \frac{\beta}{\alpha \Omega}\left(T^{\lambda \mu}-E q^{\lambda \mu}\right) \partial_{\mu} \Phi .
$$

Finally, the equation of motion for the gravitational field $\Phi$ takes the form:

$$
\sqrt{V} \square \Phi=\kappa \chi,
$$

where

$$
\chi=\frac{1}{2}\left(\frac{\alpha^{\prime}}{2 \alpha}(T-E)+\frac{Z^{\prime}}{2 Z} E-\nabla_{\lambda} C^{\lambda}\right) .
$$

Substituting the value $\alpha=e^{-2 \Phi}$ and using the equation (23) for the potential $V$ we rewrite this expression under the form

$$
\chi=\frac{1}{2}\left(\frac{3 e^{2 \Phi}+1}{3 e^{2 \Phi}-1} E-T-\nabla_{\lambda} C^{\lambda}\right) .
$$

This equation describes the dynamics of our GSG in presence of matter, under the assumptions (12) and (23). The quantity $\chi$ involves a non-trivial coupling between the gradient of the scalar field $\nabla_{\mu} \Phi$ and the complete energy-momentum tensor of the matter field $T_{\mu \nu}$ and not uniquely its trace. This property allows the electromagnetic field to interact with the gravitational field. The Newtonian limit gives the identification

$$
\kappa \equiv \frac{8 \pi G}{c^{4}} .
$$




\section{Natural decomposition}

The form of the metric, containing the derivative of $\Phi$ suggests a simplification in the description of the matter terms which is useful for exploring the cosmological consequences of GSG. Suppose that $\partial_{\mu} \Phi$ is time-like, that is $\Omega>0$. We then define the normalized vector

$$
I_{\mu}=\frac{\partial_{\mu} \Phi}{\sqrt{\Omega}}
$$

This vector can be used to decompose the energymomentum tensor of a perfect fluid in the "co-moving" representation by setting

$$
T^{\mu \nu}=(\varrho+p) I^{\mu} I^{\nu}-p q^{\mu \nu},
$$

it then follows

$$
T^{\mu \nu} \partial_{\mu} \Phi=\sqrt{\Omega} \varrho I^{\mu},
$$

and

$$
T^{\mu \nu} \partial_{\mu} \Phi \partial_{\nu} \Phi=\Omega \varrho
$$

Thus, in this frame the quantities $E$ and $T$ reduces to

$$
E=\varrho, \quad T=\varrho-3 p .
$$

Using these results it follows that $C^{\mu}=0$. In the natural frame associated to the gradient of the gravitational field $\Phi$ the equation of motion for the scalar gravity, reduces to the form

$$
\sqrt{V} \square \Phi=-\frac{\kappa}{2}\left(\frac{2 \alpha}{\alpha-3} \varrho-3 p\right) .
$$

This is the form of the dynamics of $\Phi$ when the source is a perfect fluid. In the next section we provide a simple example of GSG in the analysis of the global properties of the universe.

\section{COSMOLOGY}

In this section we start discussing some cosmological aspects of Geometric Scalar Gravity.

We work in a Gaussian coordinate system that provides a $3+1$ decomposition of the spacetime manifold. Because of homogeneity and isotropy, the gravitational field depends only on the global time T:

$$
\Phi=\Phi(T)
$$

We set for the auxiliary (non-observable) Minkowski background the expression

$$
d s_{M}^{2}=d T^{2}-d x^{2}-d y^{2}-d z^{2}
$$

Then the gravitational metric is given by

$$
q^{00}=Z, q^{11}=q^{22}=q^{33}=-\alpha,
$$

where $\alpha=\alpha(T)$ and $Z=Z(T)$.Then the gravitational metric takes the form

$$
d s^{2}=\frac{1}{Z} d T^{2}-\frac{1}{\alpha}\left(d x^{2}+d y^{2}+d z^{2}\right) .
$$

After making some redefinitions $\alpha \equiv 1 / a^{2}$ and $d t=$ $d T / \sqrt{Z}$, the line element reduces to

$$
d s^{2}=d t^{2}-a(t)^{2}\left(d x^{2}+d y^{2}+d z^{2}\right) .
$$

We use the natural frame defined in the previous section to write the source of the gravitational field as a perfect fluid with equation of state $p=\lambda \rho$. Using Eq. (12) it follows that $a=e^{\Phi}$. Therefore, the dynamical equation for the gravitational field $\Phi$ becomes an equation of motion in terms of the scale factor $a(t)$ :

$$
\sqrt{V}\left(\frac{\ddot{a}}{a}+2 \frac{\dot{a}^{2}}{a^{2}}\right)=-\frac{\kappa}{2}\left(\frac{2}{1-3 a^{2}} \varrho-3 p\right),
$$

where $\dot{X} \equiv d X / d t$. On the other hand, the equation of conservation of the energy-momentum tensor yields

$$
\varrho=\varrho_{0} a^{-3(1+\lambda)} .
$$

Using this formula in Eq. (41), yields

$$
\frac{2}{\kappa} \sqrt{V}\left(\frac{\ddot{a}}{a}+2 \frac{\dot{a}^{2}}{a^{2}}\right)=-\frac{2-3 \lambda+9 \lambda a^{2}}{1-3 a^{2}} \varrho_{0} a^{-3(1+\lambda)} .
$$

We should compare this behavior of the scalar factor of the cosmological metric with the corresponding equations obtained in general relativity. In GR there are two equations. A dynamical one

$$
\frac{\ddot{A}}{A}=-\frac{k}{6}(1+3 \lambda) \varrho,
$$

and a constraint

$$
\left(\frac{\dot{A}}{A}\right)^{2}=\frac{k}{3} \varrho
$$

where $A$ plays the role of $a(t)$ in these equations.

Equation (44) corresponds to the true dynamical one and the other one is nothing but restricts the initial condition. Therefore, the integration constants of Eq. (44) are not arbitrary. There is no analogue constraint equation in GSG. We postpone the further analysis of the cosmological description of the scalar gravity for a future work. 


\section{CONCLUSION AND OUTLOOK}

Before proposing to jump from one to ten quantities for describing the gravitational field, Einstein and other contemporary researchers tried the natural modification from one function as used in Newtonian gravity to one scalar (special relativistic) field. However, all these attempts failed.

Einstein then came with his beautiful idea that gravity is a metrical phenomenon and the jump from one to ten functions became natural. Besides, the success of general relativity made this excess of variables not a weak point but, on the contrary, it appeared as necessary.

In the present paper, we have clarified many facets of the problem and showed how it is possible to construct a metrical theory of gravity (Einstein's insight) avoiding the necessity to increase the number of fundamental variables in the Geometrical Scalar Gravity.

Our proposal deals with gravitational processes through a scalar field in a natural extension of Newton's theory. The traditional drawbacks of scalar gravity are overcome. The auxiliary Minkowski geometry $\eta_{\mu \nu}$ is not observable. This procedure is similar to the background field formulation of General Relativity proposed by Gupta, Feynman, Deser, Grischuk and others. Matter interacts with the field $\Phi$ only through the gravitational metric

$$
q^{\mu \nu}=\alpha \eta^{\mu \nu}+\frac{\beta}{w} \partial^{\mu} \Phi \partial^{\nu} \Phi
$$

where

$$
\begin{gathered}
\alpha=e^{-2 \Phi}, \\
\beta=\alpha\left(\alpha^{2} V-1\right) .
\end{gathered}
$$

The fundamental Lemma relates a class of dynamical systems described by the Lagrangian $L=V(\Phi) w$ to a massless Klein-Gordon self-interacting field in a curved spacetime.

The self-interaction of the scalar field is described by the potential $V(\Phi)$ which acts multiplicatively on the kinetic term. Different forms of $V(\Phi)$ yields different gravitational theories. In the present paper we analyzed the case in which

$$
V=\frac{(\alpha-3)^{2}}{4 \alpha^{3}}
$$

Such particular choice allows solutions in agreement with actual observational knowledge of gravity (see appendix $\mathrm{C})$. The crucial issue of gravitational radiation and in particular the emission of gravitational energy from the compact sources will be analyzed in a future paper.

In appendix A, we exhibit a preliminary analysis based on Ref. [15], in which we show that the rate of energy emitted by a monopole radiation in our GSG has the same order of magnitude of the quadrupole radiation in general relativity. This and the cosmological consequences of Geometric Scalar Gravity deserve a more specific analysis that will be described elsewhere.

\section{APPENDIX A: GRAVITATIONAL RADIATION}

As mentioned before, a theory of gravity should be prepared to account for the observed behavior of binary pulsars as a consequence of the emission of gravitational energy (for instance, see [16]). General relativity makes use of the quadrupole radiation formula with success. Let us analyze this phenomenon from the point of view of GSG.

We follow the main lines as presented in [15] and calculate the equation of motion of the scalar field $\Phi$ in the Minkowski background under the weak field regime. We provide then an estimative of the order of magnitude of the gravitational waves in the radiation zone using GSG theory.

We bound the discussion to the simplest case of a perfect fluid in the natural frame. This hypothesis implies that $C^{\mu}=0$. Under this consideration, the equation of motion is

$$
\sqrt{V} \square \Phi=-\frac{\kappa}{2}\left(\frac{2 \alpha}{\alpha-3} \varrho-3 p\right) .
$$

Use of the Lemma allows to rewrite Eq. (45) in the Minkowski background as follows

$$
\square_{M} \Phi+\frac{1}{2} \frac{V^{\prime}}{V} w=\frac{k}{2}\left(\frac{1}{\alpha \sqrt{V}}\right)^{3}\left(3 p-\frac{2 \alpha}{\alpha-3} \varrho\right) .
$$

Let us assume that the source of the gravitational field is dust $(p=0)$ and rewrite the energy density in terms of background quantities $\varrho=\alpha \varrho_{b}$ where $\varrho_{b}$ is the energy density in the Minkowski space-time. Inserting the expressions of $V$ and $\alpha$ in terms of $\Phi$ the equation of motion for the gravitational field is

$$
\square_{M} \Phi=\frac{k}{2}\left[\frac{2}{k} \frac{\alpha-9}{\alpha-3} w-2\left(\frac{1}{\alpha \sqrt{V}}\right)^{3} \frac{\alpha^{2}}{\alpha-3} \varrho_{b}\right] .
$$

This equation of motion has an extra term proportional to $w$ which involves first derivatives of the scalar field. To proceed and calculate the total rate of energy emission for a spherically symmetric energy distribution, we have to take into account conservation laws. Matter conservation implies

$$
M_{0}=\int d^{3} x \gamma \varrho_{b}=\text { const }
$$


where $\gamma \equiv\left[1-(v / c)^{2}\right]^{-1}$ is the Lorentz factor. This formula is responsible to the non-radiative term of the scalar field as we shall see.

The energy-momentum tensor of the scalar field in the Minkowski background is given by

$$
T_{\Phi}^{\mu \nu}=\frac{2 V}{k}\left(\partial^{\mu} \Phi \partial^{\nu} \Phi-\frac{\omega}{2} \eta^{\mu \nu}\right) .
$$

This expression will be useful to calculate the rate of radiative emission. By applying the Green method to solve Eq. (47), the scalar field $\Phi$ takes the form

$$
\Phi(t, \mathbf{x})=-G \int d^{3} x^{\prime} \frac{[\tilde{\varrho}]_{r e t}}{\left|\mathbf{x}-\mathbf{x}^{\prime}\right|},
$$

where $[X]_{\text {ret }}$ means the evaluation of $X$ in the retarded time $t-\left|\mathbf{x}-\mathbf{x}^{\prime}\right|$ and

$$
\tilde{\varrho} \equiv\left[-\frac{2}{k} \frac{\alpha-9}{\alpha-3} w+2\left(\frac{1}{\alpha \sqrt{V}}\right)^{3} \frac{\alpha^{2}}{\alpha-3} \varrho_{b}\right] .
$$

We define the rest density by

$$
\varrho_{0} \equiv \gamma \varrho_{b}
$$

Then, in the wave zone regime $\left(r=|\mathbf{x}|\right.$ and $\left.r \approx r^{\prime} \cos \theta^{\prime}\right)$, we get

$$
\begin{aligned}
\Phi(t, \mathbf{x})= & -\frac{G}{r}\left(\int d^{3} x^{\prime}\left[2\left(\frac{1}{\alpha \sqrt{V}}\right)^{3} \frac{\alpha^{2}}{\alpha-3} \frac{\varrho_{0}}{\gamma}\right]_{r e t}+\right. \\
& \left.-\frac{2}{k} \int d^{3} x^{\prime}\left[\frac{\alpha-9}{\alpha-3}\left(\dot{\Phi}^{2}-(\nabla \Phi)^{2}\right)\right]_{r e t}\right)+\ldots
\end{aligned}
$$

Due to the partial derivatives of the scalar field, the second term on the right hand side of this equation is of second order in $\Phi$ and can be dropped out. Expanding the remaining term in Taylor series, we obtain

$$
\begin{aligned}
\varrho_{0}\left(t^{\prime}, \mathbf{x}^{\prime}\right)= & \varrho_{0}\left(t-r, \mathbf{x}^{\prime}\right)+r^{\prime} \cos \theta^{\prime} \varrho_{0}+ \\
& +\frac{1}{2} r^{\prime 2} \cos ^{2} \theta^{\prime} \ddot{\varrho}_{0}+\ldots
\end{aligned}
$$

and

$$
\frac{2}{\gamma}\left(\frac{1}{\alpha \sqrt{V}}\right)^{3} \frac{\alpha^{2}}{\alpha-3}=-\left[1-11 \Phi-v^{2}\right]_{t-r}+\ldots
$$

Therefore, the leading-order radiative term in the spherically symmetric case is

$$
\Phi(t, r)=-\frac{4 \pi G}{r}\left\{\int d r^{\prime} r^{2}\left[\varrho_{0}\left(11 \Phi+v^{2}\right)\right]_{r e t}+\mathcal{O}(2)\right\},
$$

Now we use Eq. (55) and the virial relation $v^{2} \sim$ $G M / R$ to estimate $\Phi$ for a source of mass $M$, radius $R$ and velocity $v$, in the weak-field regime:

$$
\Phi \sim \frac{G M}{r c}\left(\frac{v}{c}\right)^{2} .
$$

The definition of the total rate of energy emission in the wave zone is given by

$$
\frac{d \mathcal{E}}{d t}=-4 \pi r^{2} T_{\Phi}^{0 r} \approx-\frac{1}{G}(r \dot{\Phi})^{2},
$$

where we use $d \Phi / d r=-d \Phi / d t$ and Eq. (49) to express the radiative energy flux

$$
T_{\Phi}^{0 r}=\frac{1}{4 \pi G} \dot{\Phi}^{2} .
$$

Finally, using the correct units, we get

$$
\frac{d \mathcal{E}}{d t} \sim \frac{c}{G} \Phi^{2} \sim \frac{c}{G}\left(\frac{G M v^{2}}{r c^{3}}\right)^{2} \sim \frac{G}{c^{5}}\left(\frac{M v^{2}}{r}\right)^{2} .
$$

It is clear that a very careful analysis on this topic should be done. The aim of this section is just to show that the non-linear scalar theory of gravity presented here provides a monopole radiation for a spherically symmetric mass distribution in the GSG of the same order of magnitude of the quadrupole gravitational radiation $G / c^{5}$ as in general relativity. A more precise evaluation of the binary pulsar timing is a non-trivial matter which we intend to do in the near future.

\section{APPENDIX B: Hadamard Discontinuity}

Let us analyze the discontinuities of the electromagnetic field in our scalar theory of gravity. We shall show that the electromagnetic waves will follow a geodesic motion in $q_{\mu \nu}$.

We proceed according to the standard Hadamard method (see details in 17 and references therein) and obtain the dispersion relation of the waves. Let $\Sigma$ be a surface of discontinuity of the field $A_{\mu}$. The discontinuity of an arbitrary function $f$ is given by:

$$
[f(x)]_{\Sigma}=\lim _{\epsilon \rightarrow 0^{+}}(f(x+\epsilon)-f(x-\epsilon)) .
$$

The field $A_{\mu}$ and its first derivative $\partial_{\nu} A_{\mu}$ are continuous across $\Sigma$, while the second derivatives presents a discontinuity:

$$
\begin{aligned}
{\left[A_{\mu}\right]_{\Sigma} } & =0 \\
{\left[\partial_{\nu} A_{\mu}\right]_{\Sigma} } & =0 \\
{\left[\partial_{\alpha} \partial_{\beta} A_{\mu}\right]_{\Sigma} } & =k_{\alpha} k_{\beta} \xi_{\mu}(x),
\end{aligned}
$$


where $k_{\mu} \equiv \partial_{\mu} \Sigma$ is the propagation vector and $\xi_{\mu}(x)$ the amplitude of the discontinuity. Using these discontinuity properties in the equation of motion

$$
q^{\alpha \nu} A_{[\mu, \nu] ; \alpha}=0,
$$

it follows that:

$$
k_{\alpha} k_{\beta} q^{\alpha \beta}=0 .
$$

This means that the discontinuities of the electromagnetic field propagate as null geodesics in the metric $q_{\mu \nu}$. It happens due to the relation between the EM field and the metric, which is a minimal coupling from the general relativity point of view. Therefore, any other kind of matter will see only the metric $q_{\mu \nu}$.

\section{APPENDIX C: The form of the potential V}

From the relation imposed by the Lemma

$$
V=\frac{Z}{\alpha^{3}},
$$

it follows that the observation of $Z$ specifies the potential. From equation (21) it follows that

$$
-q_{11} \equiv B=\frac{\alpha(\alpha-3)^{2}}{4 Z} .
$$

Observations have been made up to second order on $x \equiv r_{H} / r$. This means that we can be confident that up to this order the form of the $q_{11}$ coefficient of the gravitational metric is given by

$$
B \approx 1+x+x^{2}
$$

This implies that one can be sure on the expansion of $Z$ up to second order on $\alpha$. Any further dependence on $\alpha$ should be impossible to be univocally fixed in this order of expansion. Indeed, if we set

$$
Z=M \alpha^{2}+N \alpha+P,
$$

substituting in this expression and expanding all quantities up to second order $O\left(x^{2}\right)$ and noting that

$$
q_{00}=\frac{1}{\alpha}=1-x
$$

yields

$$
\alpha \approx 1+x+x^{2}
$$

it follows

$$
M=\frac{1}{4} ; \quad N=-\frac{3}{2} ; \quad P=\frac{9}{4} .
$$

In other words, this gives

$$
Z=\frac{(\alpha-3)^{2}}{4}
$$

and consequently

$$
V=\frac{(\alpha-3)^{2}}{4 \alpha^{3}}
$$

This corresponds to the expression (23).

\section{APPENDIX D: Geometrical version of Einstein-Nordström theory}

Let us consider now the particular case in which the potential takes the form

$$
V=\frac{1}{\alpha^{2}}
$$

and set

$$
\alpha=e^{2 \Phi}
$$

As a consequence, the coefficient $\beta$ of the metric $q_{\mu \nu}$ vanishes. The form of the metric reduces to a conformally flat geometry, that is

$$
q_{\mu \nu}=e^{-2 \Phi} \eta_{\mu \nu}
$$

The equation of motion (33) is the Einstein-Nordström dynamics

$$
\square \Phi=-\frac{\kappa}{2} e^{2 \Phi} T
$$

that is

$$
\square \Phi=-\frac{\kappa}{2} T^{\mu \nu} \eta_{\mu \nu}
$$

once $T=T^{\mu \nu} q_{\mu \nu}=T^{\mu \nu} e^{-2 \Phi} \eta_{\mu \nu}$. A direct comparison with the limit of weak field as presented in a previous section shows that this is not a good proposal once it describes configurations which are not compatible with the weak gravitational field. The theory constructed with such bad choice of the potential $V$ is nothing but the unsuccessful Einstein-Nordström scalar gravity.

\section{Acknowledgements}

MN and EB would like to thank ICRANet (Pescara) and Università degli Studi dell'Insubria (Como) for their hospitality where part of this paper was written. This work was supported by Conselho Nacional de Desenvolvimento Científico e Tecnológico (CNPq), FINEP and Fundação de Amparo à Pesquisa do Estado do Rio de Janeiro (FAPERJ) of Brazil. 
[1] G. Gibbons and C. Will, On the multiple deaths of Whitehead's theory of gravity, arXiv:gr-qc/0611006 1 (2006).

[2] D. Giulini, What is (not) wrong with scalar gravity?, arXiv:gr-qc/0611100v2 (2008).

[3] C. Will, The Confrontation between General Relativity and Experiment, Liv. Rev. Rel. 93 (2006).

[4] J. Stachel et al., editors, The collected papers of Albert Einstein, vol. 1-9, Princeton University Press, Princeton, New Jersey (1987-2005);

[5] S. N. Gupta Einstein's and others theories of gravitation, Phys. Rev. 961683 (1954).

[6] R. P. Feynman, F. B. Morinigo and W. G. Wagner, Feynman lectures on gravitation, Addison Wesley Pub. Company, Massachusetts, (1995).

[7] S. Deser, Gravity from self-interaction in a curved background, Class. Quantum Grav. 4 L99 (1987).

[8] J. P. Grishchuk, A. N. Petrov and A. D. Popova, Exact theory of the (Einstein) gravitational field in an arbitrary background space-time, Commun. Math. Phys. 94 379 (1984).

[9] M. Novello and E. Goulart, Beyond analog gravity: the case of exceptional dynamics, Class. Quantum Grav. 28 145022 (2011).
[10] J. Magueijo, Phys Rev D 79043525 (2009).

[11] E. Goulart, M. Novello, F. T. Falciano and J. D. Toniato, Hidden geometries in nonlinear theories: a novel aspect of analogue gravity, Class. Quantum Grav. 28245008 (2011).

[12] A. Einstein, The meaning of relativity, Princeton University Press, (1950).

[13] G. Nordström, Phys. Zeit. 131126 (1912).

[14] F. Ravndal, Scalar gravitation and extra dimensions, arXiv:gr-qc/0405030 1 (2004).

[15] S. L. Shapiro and S. A. Teukolsky, Scalar gravitation: A laboratory for numerical relativity, Phys. Rev. D 471529 (1993).

[16] J. H. Taylor and J. M. Weisberg, A new test of general relativity - Gravitational radiation and the binary pulsar PSR 1913+16, Astroph. J. 253908 (1982); J. H. Taylor and J. M. Weisberg, Further experimental tests of relativistic gravity using the binary pulsar PSR $1913+16$, Astroph. J. 345434 (1989).

[17] M. Novello, V, De Lorenci, R. Klippert and J M Salim Geometrical aspects of light propagation in non-linear electrodynamics, Phys. Rev D 61, 045001 (2000). 